\title{
Artificial pancreas treatment for outpatients with type 1 diabetes: systematic review and meta-analysis
}

\author{
Eleni Bekiari, ${ }^{1}$ Konstantinos Kitsios, ${ }^{2}$ Hood Thabit, ${ }^{3}$ Martin Tauschmann, ${ }^{3}$ Eleni Athanasiadou, \\ Thomas Karagiannis, ${ }^{1}$ Anna-Bettina Haidich, ${ }^{4}$ Roman Hovorka, ${ }^{3}$ Apostolos Tsapas ${ }^{1,5}$
}

$\overline{{ }^{1} \text { Clinical Research and Evidence }}$ Based Medicine Unit, Aristotle University of Thessaloniki, 54642 Thessaloniki, Greece

${ }^{2}$ Diabetes Centre, Second Medical Department, Aristotle University of Thessaloniki, Thessaloniki, Greece

${ }^{3}$ Wellcome Trust-Medical Research Council Institute of Metabolic Science, University of Cambridge, Cambridge, UK ${ }^{4}$ Department of Hygiene and Epidemiology, Medical School, Aristotle University of Thessaloniki, Thessaloniki, Greece

${ }^{5}$ Harris Manchester College, University of Oxford, Oxford, UK Correspondence to: A Tsapas atsapas@auth.gr

Additional material is published online only. To view please visit the journal online.

Cite this as: $B M J$ 2018;361:k1310 http://dx.doi.org/10.1136/bmj.k1310

Accepted: 2 March 2018

\section{ABSTRACT}

OBJECTIVE

To evaluate the efficacy and safety of artificial pancreas treatment in non-pregnant outpatients with type 1 diabetes.

\section{DESIGN}

Systematic review and meta-analysis of randomised controlled trials.

\section{DATA SOURCES}

Medline, Embase, Cochrane Library, and grey literature up to 2 February 2018.

\section{ELIGIBILITY CRITERIA FOR SELECTING STUDIES}

Randomised controlled trials in non-pregnant outpatients with type 1 diabetes that compared the use of any artificial pancreas system with any type of insulin based treatment. Primary outcome was proportion (\%) of time that sensor glucose level was within the near normoglycaemic range (3.9-10 $\mathrm{mmol} / \mathrm{L})$. Secondary outcomes included proportion (\%) of time that sensor glucose level was above 10 $\mathrm{mmol} / \mathrm{L}$ or below $3.9 \mathrm{mmol} / \mathrm{L}$, low blood glucose index overnight, mean sensor glucose level, total daily insulin needs, and glycated haemoglobin. The Cochrane Collaboration risk of bias tool was used to assess study quality.

RESULTS

40 studies (1027 participants with data for 44 comparisons) were included in the meta-analysis. 35 comparisons assessed a single hormone artificial pancreas system, whereas nine comparisons

\section{WHAT IS ALREADY KNOWN ON THIS TOPIC}

Individual studies have shown artificial pancreas use to be safe and efficacious in inpatients, patients under close monitoring, and outpatients with type 1 diabetes

The US Food and Drug Administration recently approved artificial pancreas use for patients aged 14 years and older with type 1 diabetes

Previous meta-analyses on artificial pancreas systems have provided limited findings, mainly owing to the low number of studies incorporated and heterogeneous definitions of outcomes

\section{WHAT THIS STUDY ADDS}

In view of all the available evidence from randomised controlled trials, artificial pancreas treatment significantly improves glycaemic control while reducing the burden of hypoglycaemia in outpatients with type 1 diabetes

Results are consistent for people using artificial pancreas systems unsupervised under normal living conditions, and for both single hormone and dual hormone systems

The current research evidence on artificial pancreas systems is limited by inconsistency in outcome reporting, small sample size, and short follow-up duration of individual trials assessed a dual hormone system. Only nine studies were at low risk of bias. Proportion of time in the near normoglycaemic range $(3.9-10.0 \mathrm{mmol} / \mathrm{L})$ was significantly higher with artificial pancreas use, both overnight (weighted mean difference 15.15\%, 95\% confidence interval $12.21 \%$ to $18.09 \%$ ) and over a 24 hour period (9.62\%, $7.54 \%$ to $11.7 \%)$. Artificial pancreas systems had a favourable effect on the proportion of time with sensor glucose level above $10 \mathrm{mmol} / \mathrm{L}(-8.52 \%,-11.14 \%$ to $-5.9 \%)$ or below $3.9 \mathrm{mmol} / \mathrm{L}(-1.49 \%,-1.86 \%$ to $-1.11 \%)$ over 24 hours, compared with control treatment. Robustness of findings for the primary outcome was verified in sensitivity analyses, by including only trials at low risk of bias (11.64\%, $9.1 \%$ to $14.18 \%)$ or trials under unsupervised, normal living conditions $(10.42 \%$, $8.63 \%$ to $12.2 \%$ ). Results were consistent in a subgroup analysis both for single hormone and dual hormone artificial pancreas systems.

\section{CONCLUSIONS}

Artificial pancreas systems are an efficacious and safe approach for treating outpatients with type 1 diabetes. The main limitations of current research evidence on artificial pancreas systems are related to inconsistency in outcome reporting, small sample size, and short follow-up duration of individual trials.

\section{Introduction}

Despite substantial advances in the treatment of type 1 diabetes, maintaining good glycaemic control without hypoglycaemia remains a challenge for patients at all ages and for healthcare providers. Currently, insulin treatment strategies in type 1 diabetes include either multiple daily insulin injections or continuous subcutaneous insulin infusion with an insulin pump. In 2008, the National Institute for Health and Care Excellence concluded that continuous subcutaneous insulin infusion has a favourable effect on glycated haemoglobin $\left(\mathrm{HbA}_{1 \mathrm{c}}\right)$ and incidence of hypoglycaemia in patients with type 1 diabetes. ${ }^{1}$ Moreover, a metaanalysis of 19 trials concluded that continuous subcutaneous insulin infusion had a favourable effect on glycaemic control in adults with type 1 diabetes compared with multiple daily insulin injections. ${ }^{2}$ In addition, in a recent cluster randomised controlled trial, patients with type 1 diabetes who used continuous subcutaneous insulin infusion instead of multiple daily insulin injections reported additional benefits in quality of life and greater treatment satisfaction. ${ }^{3}$

Until recently, continuous subcutaneous insulin infusion was mostly guided by self-monitoring of capillary glucose testing. ${ }^{4}$ However, insulin pumps are now also used in conjunction with real time continuous glucose monitoring, hence allowing the 
patient to manually modify the insulin infusion rate according to continuous glucose monitoring values (known as sensor augmented pump treatment). ${ }^{45}$ The recent introduction of a low glucose suspend feature has allowed for automatic pump suspension when a preprogrammed threshold value of continuous glucose monitoring is reached. ${ }^{6}$ Based on a 2016 analysis, the use of sensor augmented pump treatment and the low glucose suspend feature was found to be cost effective compared with continuous subcutaneous insulin infusion and self-monitoring of blood glucose for patients with type 1 diabetes in the United Kingdom. ${ }^{7}$

Artificial pancreas treatment, also referred to as closed loop glucose control, is an emerging treatment option combining an insulin pump and continuous glucose monitoring with a control algorithm to deliver insulin in a glucose responsive manner (that is, a single hormone artificial pancreas system). Glucagon can also be delivered in a similar glucose responsive fashion, as accommodated by dual hormone artificial pancreas systems. Therefore, compared with insulin pumps or sensor augmented pumps, artificial pancreas use can reduce the burden for patients by automatically adjusting the amount of insulin entering the body on the basis of sensor glucose levels. Several artificial pancreas systems have been developed, and their safety and efficacy have been evaluated in many studies, showing promising results. An early pooled analysis included only four studies in an inpatient setting, ${ }^{8}$ whereas an overview published in 2015 summarised existing data from randomised controlled trials up to September 2014. ${ }^{9}$ Finally, a recent metaanalysis summarised evidence from published trials of artificial pancreas systems in outpatients with type 1 diabetes. ${ }^{10}$ Notably, the US Food and Drug Administration has recently approved the first artificial pancreas system for use by people with type 1 diabetes over 14 years of age, based on a safety outpatient study. ${ }^{11}$ This systematic review and metaanalysis aimed to summarise and critically appraise all existing evidence on the clinical efficacy and safety of artificial pancreas systems for the management of type 1 diabetes in the outpatient setting.

\section{Methods}

This systematic review and meta-analysis is based on a prespecified protocol (appendix 1), and is reported according to the preferred reporting items for systematic reviews and meta-analyses (PRISMA) statement (appendix 2). ${ }^{12}$

\section{Search strategy and selection criteria}

We searched Medline, Embase, Cochrane Database of Systematic Reviews, and the Central Register of Controlled Trials from inception to 2 February 2018. Our search strategy was based on search terms describing the intervention (artificial pancreas or closed loop system) in addition to a filter for randomised trials. We omitted terms related to type 1 diabetes to avoid missing potentially relevant studies. ${ }^{13}{ }^{14} \mathrm{We}$ used search terms that had been identified from initial scoping searches, target references, and browsing of database thesauruses (web appendix 3). We imposed no restrictions based on language or publication status, searched ClinicalTrials.gov, and sought for additional studies from snowballing of included records.

We included randomised controlled trials in nonpregnant adults, children, and adolescents with type 1 diabetes in the outpatient setting (including hotels, diabetes camps, or normal living conditions), irrespective of trial design (parallel or crossover) or duration of intervention, which compared artificial pancreas systems with any type of insulin based treatment. Such comparative treatments included multiple daily insulin injections, insulin pump treatment without continuous glucose monitoring or with blinded continuous glucoses monitoring, and sensor augmented pumps with or without a low glucose suspend feature.

\section{Data extraction}

References identified were imported into a reference management software (Endnote, Clarivate Analytics) for deduplication. Potentially eligible records were exported to Covidence (Covidence, Veritas Health Innovation) for screening. Three reviewers (EB, EA, and $\mathrm{KK}$ ) working independently, screened all records in duplicate, and disagreements were arbitrated by a senior team member (AT). Initially, records were screened at title and abstract level, and potentially eligible studies were assessed in full text.

If multiple records of one study were retrieved, we collated data from all records, and used data from the report with the longest duration of follow-up. We extracted data for study and participant baseline characteristics, interventions, comparators, and clinical outcomes in duplicate (EB, EA, and TK) by using an electronic, pilot tested, data extraction form (web appendix 4). Disagreements were resolved by consensus or following discussion with a senior reviewer (AT).

\section{Outcomes}

The primary outcome was proportion (\%) of time when the sensor glucose level was within the near normoglycaemic range $(3.9-10 \mathrm{mmol} / \mathrm{L})$. Secondary outcomes included proportion (\%) of time when the sensor glucose level was above $10 \mathrm{mmol} / \mathrm{L}$ or below $3.9 \mathrm{mmol} / \mathrm{L}$, incidence of severe hypoglycaemia, mean sensor glucose level, total daily insulin needs, and glycated haemoglobin $\left(\mathrm{HbA}_{1 \mathrm{c}}\right)$. We also used low blood glucose index overnight as an additional outcome to assess hypoglycaemia. Low blood glucose index is a weighted average of the number of hypoglycaemic readings with progressively increasing weights as glucose levels decrease and is associated with the risk of hypoglycaemia and prediction of severe hypoglycaemic episodes. ${ }^{15}$ When available, for proportion (\%) of time in the near normoglycaemic range, hyperglycaemia ( $>10 \mathrm{mmol} / \mathrm{L})$, or hypoglycaemia ( $<3.9 \mathrm{mmol} / \mathrm{L})$, we extracted data both for 24 hour and overnight periods (as defined in each individual study). 


\section{Statistical analysis}

We conducted meta-analyses when data were available for at least two studies. We calculated weighted mean differences with $95 \%$ confidence intervals, applying an inverse variance weighted random effects model using the DerSimonian and Laird estimation method. ${ }^{16}$ We also calculated $95 \%$ prediction intervals to estimate a predicted range for the true treatment effect in any one individual study. ${ }^{17}$ In addition, to account for uncertainty related to heterogeneity estimates, we calculated 95\% confidence intervals applying the Hartung Knapp correction method. ${ }^{18}$ For trials reporting only medians and interquartile ranges, we retrieved mean and variance values from authors of original reports or used appropriate formulas to calculate mean and variance, making no assumption on the distribution of the underlying data. ${ }^{19}$ We combined data both from parallel group and crossover studies. Finally, for crossover studies that reported their results as parallel group trials, we used appropriate methods to impute within patient differences. ${ }^{20}$

We conducted prespecified subgroup analyses based on the mode of use (overnight or over 24 hours) and type of artificial pancreas system (single or dual hormone). A series of a priori decided sensitivity analyses was conducted for the primary outcome, excluding trials at unclear or high risk of bias, trials recruiting people in diabetes camps, or trials with supervised use of artificial pancreas system. We assessed statistical heterogeneity by the $\chi^{2}$ based Cochran $\mathrm{Q}$ test and the $\tau^{2}$ and $\mathrm{I}^{2}$ statistics. For $\mathrm{HbA}_{1 c}$, we synthesised only data from trials with at least eight weeks' duration per intervention. All analyses were undertaken in RevMan 5.3 (Nordic Cochrane Centre) and Stata 13.0 (Stata Corporation).

\section{Assessment of risk of bias in individual studies and across studies}

Quality assessment was undertaken in duplicate by two independent reviewers (EB and EA), and disagreements were resolved by consensus or arbitrated by a third reviewer (AT). We used the Cochrane Collaboration risk of bias tool to assess risk of bias for the primary outcome for individual studies. For crossover studies, we also assessed a series of methodological challenges that are related to this specific design (appropriateness of crossover design, carry-over effects, unbiased data). ${ }^{21}$ We used results to provide an evaluation of the overall quality of the included studies (appendix 5) to inform a sensitivity analysis including only trials at low overall risk of bias.

We explored risk of bias across studies, both visually using a contour enhanced funnel plot, and formally using Egger's statistical test. ${ }^{22} 23$ In case of evidence of small study effects, we used the trim and fill method as a sensitivity analysis, to provide an adjusted estimate of the meta-analysis. ${ }^{24}$

\section{Patient involvement}

No patients were involved in definition of the research question or the outcome measures, and interpretation or writing up of results. Data relating to the impact of the intervention on participants' quality of life were not extracted. Where possible, results of this systematic review and meta-analysis will be disseminated to the patient community or individual patients and families through the investigators of this meta-analysis.

\section{Results}

\section{Characteristics of included studies}

Figure 1 shows the study selection process. Of 10054 records retrieved, 85 reports qualified for inclusion in our systematic review. After juxtaposing different reports that referred to the same study, 39 publications describing 41 trials (1042 participants with data for 45 comparisons) were used to inform our systematic review. $^{25-63}$ One trial did not report data for outcomes assessed and was not included in the meta-analysis. ${ }^{57}$ Table 1 shows characteristics of the 41 studies included in the systematic review and their participants at baseline. The clear majority of included trials used a crossover design, 25-27 29-35 37-41 43-46 50-58 60-63 whereas only seven trials were of parallel design. ${ }^{28} 364247-4959$ The duration of 36 trials lasted up to four weeks, ${ }^{25-41} 43$ 45-50 52-62 whereas the remaining five trials lasted from eight to 30 weeks. ${ }^{42445163}$ Seventeen trials recruited children or adolescents, $28303235363841495053-57606163$ 13 recruited adults, $25272934374043-45556263$ and 11 recruited a mixed population. ${ }^{2631394246-4851525859}$ The artificial pancreas was used overnight in 16 trials, ${ }^{29} 36$ 3839414446 50-53 57-59 6263 and used over 24 hours in the remaining 25 trials. ${ }^{25-28} 30-353740424345$ 47-49 54-56 606163 In 32 trials, a single hormone artificial pancreas system was assessed (mostly versus unblinded treatment using sensor augmented pump). ${ }^{25}$ 26 28-32 35-37 41-54 57-63

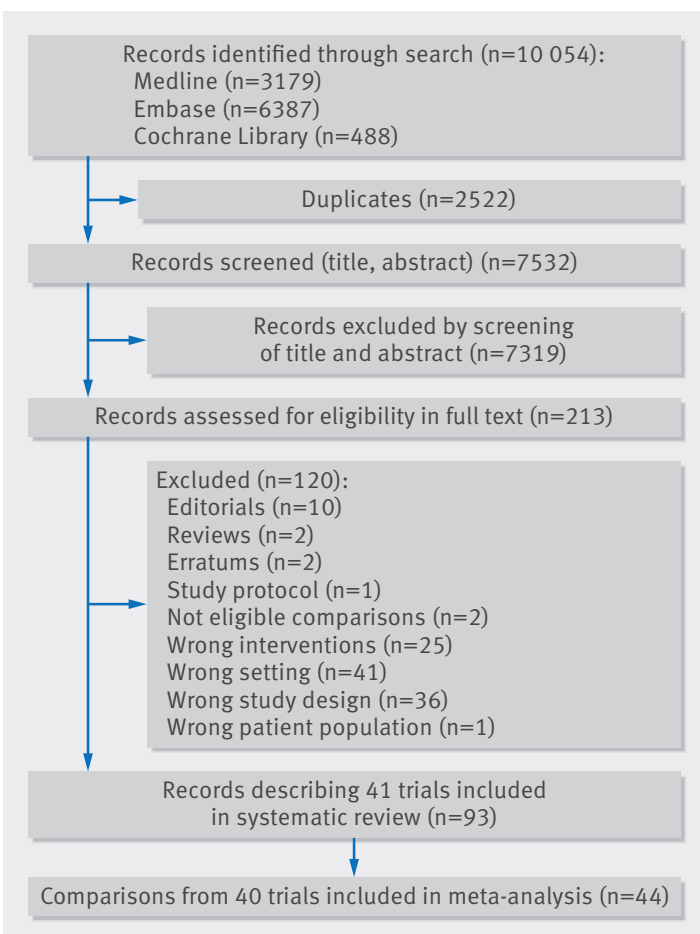

Fig 1 | Flow diagram of study selection process 


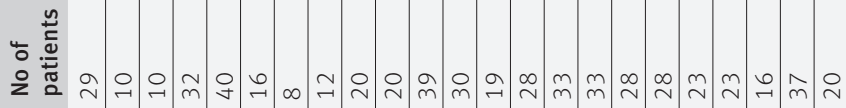 \\ lil

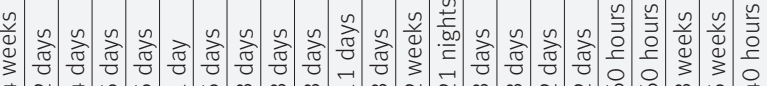

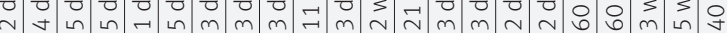

[I:

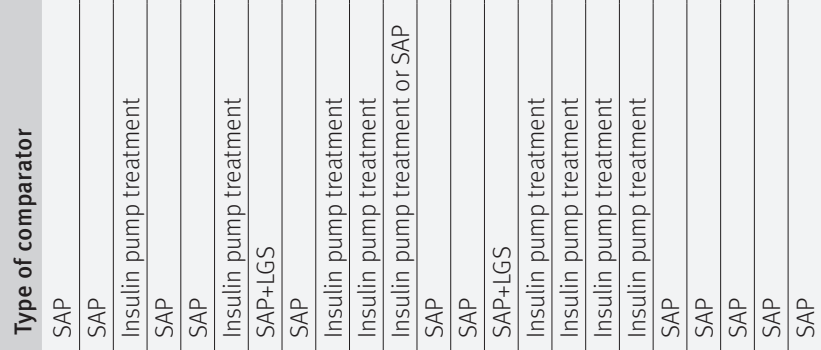

品

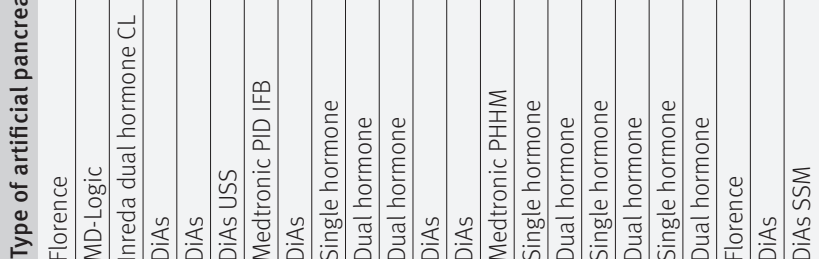

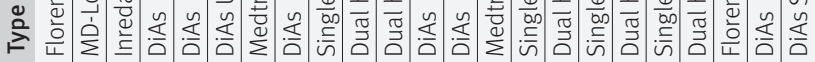

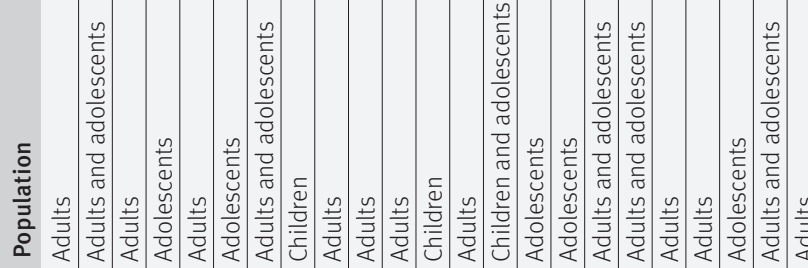

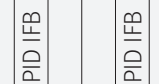

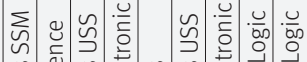

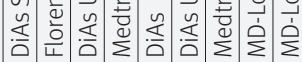

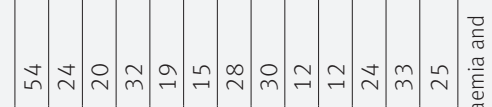

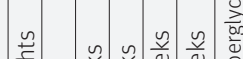

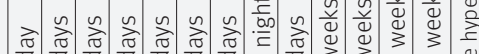

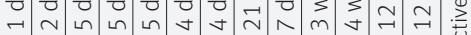

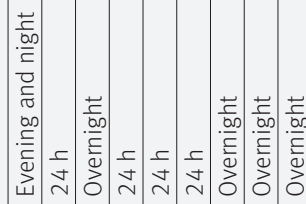

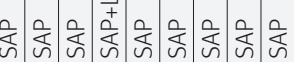

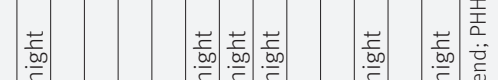

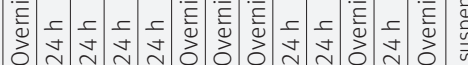
点起这 ‘े $\bar{~}$

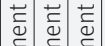

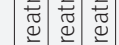

䓂

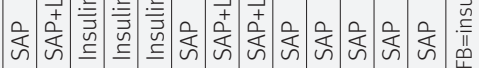

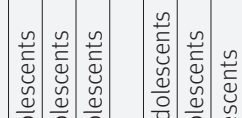

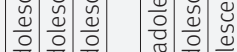

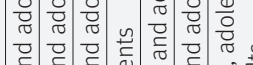

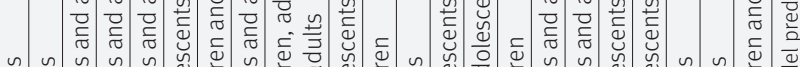

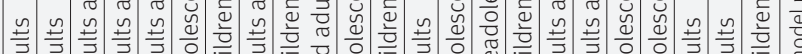

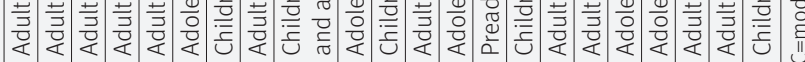

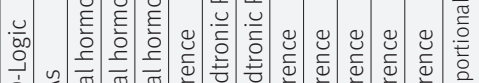

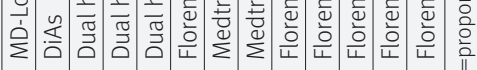

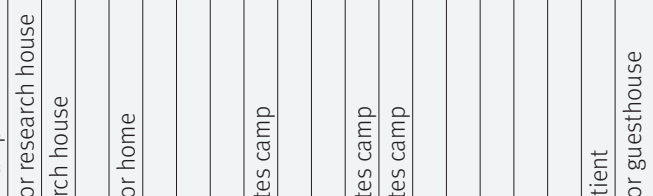

है है है है है

西

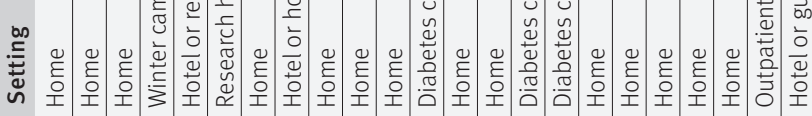

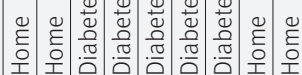

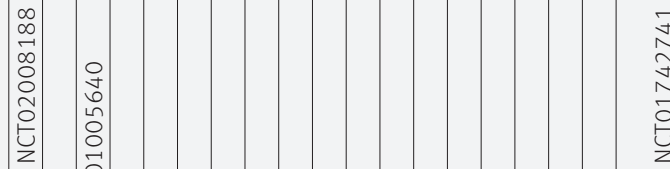

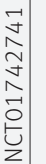

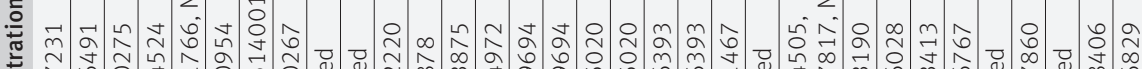

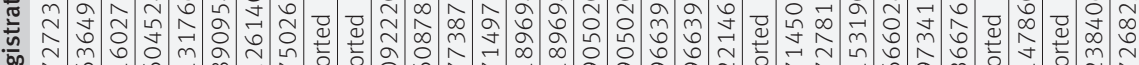

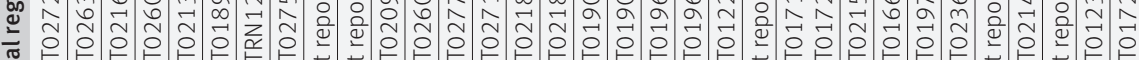

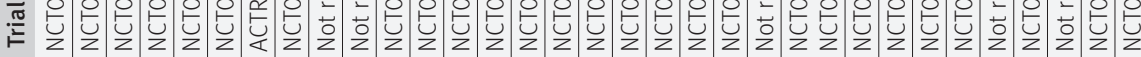

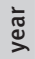

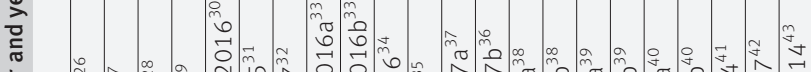

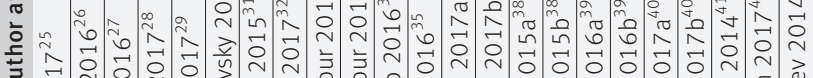

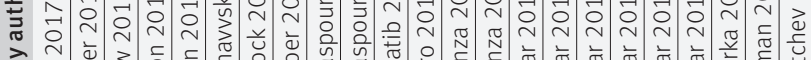

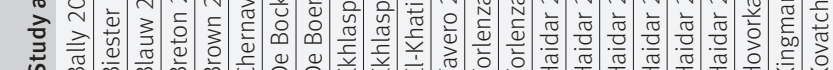

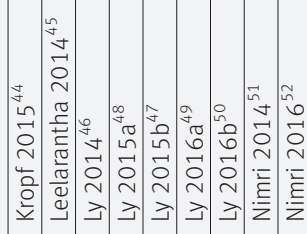

害

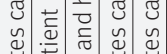

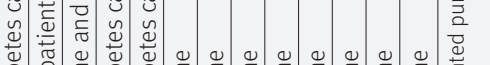

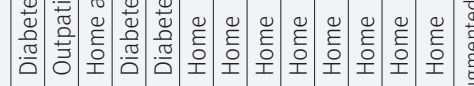

高

范高.

远最率

हों 은 월

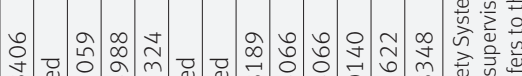

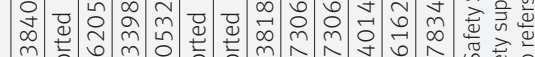

जิ

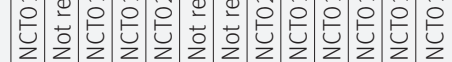

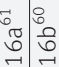

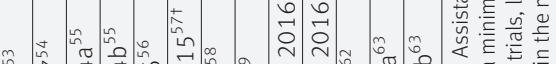
थ

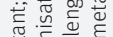

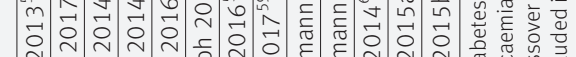

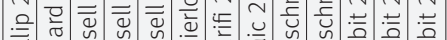

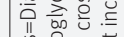

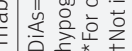


Five trials assessed a dual hormone artificial pancreas system, mainly by comparison with insulin pump treatment (consisting of continuous subcutaneous insulin infusion combined with a blinded system of continuous glucose monitoring). ${ }^{27345556}$ Additionally, four studies evaluated both a single hormone and a dual hormone system against control treatment (as three way crossover trials). ${ }^{33}$ 38-40

In six studies assessing sensor augmented pump treatment, control treatment comprised a sensor augmented pump combined with an low glucose suspend feature. ${ }^{313648545859}$ Among trials evaluating single hormone artificial pancreas systems, 13 used the DiAs platform, ${ }^{28-30} 32353742-4446474954$ eight used the Florence implementation, ${ }^{25} 41455760-63$ four used the MD-Logic platform, ${ }^{2651-53}$ and six used a Medtronic device. ${ }^{313648505859}$ Most trials used a model predictive control algorithm, ${ }^{25} 293435$ 37-41 43-45 54-57 60-63 five used a proportional integral derivative algorithm, ${ }^{27} 31485058$ four used a fuzzy logic algorithm, ${ }^{26515253}$ four used a control to range algorithm, ${ }^{30} 324649$ and the remainder used other algorithms or did not provide relevant details. 283336424759 Twenty one comparisons used a Dexcom sensor for continuous glucose monitoring, ${ }^{28-30}$ $32343537384042-4446474954-56$ while $12^{27} 31363948$ 50-53 5859 and nine 25414557 60-63 comparisons used an Enlite Sensor or a FreeStyle Navigator in the artificial pancreas systems, respectively. Type of sensor for continuous glucose monitoring was not reported in two trials. ${ }^{2633}$ In 41 comparisons, the type of sensor for continuous glucose monitoring was identical between artificial pancreas and control arms, whereas four trials did not report information for type of sensor used in the control arm. ${ }^{26304749}$

In terms of setting, 13 trials were held in a diabetes camp or a guesthouse, $282935384346-50535556$ and in 26 trials, participants were at home. $.^{25-27} \quad 30-343637$ 39-41 $444551525557-63$ Only in a small subset of trials were participants using artificial pancreas unsupervised under normal living conditions ${ }^{25} 26414560-63$; the remaining studies either used remote monitoring or did not provide relevant details. Participants' mean age and $\mathrm{HbA}_{1 \mathrm{c}}$ at baseline ranged from 7.0 to 47.0 years and from $6.9 \%$ to $8.6 \%$, respectively.

\section{Risk of bias assessment results}

Risk of bias assessment for the primary outcome is presented in appendices 6 and 7. Only nine studies were at low risk of bias. Most studies were deemed at high risk for bias, because either they reported median values instead of mean values, or reported results that required extensive use of imputation methods to be used in meta-analyses.

\section{Primary outcome}

All meta-analysis results are presented as summary effect estimates for artificial pancreas systems versus control treatment. Compared with control treatment, use of artificial pancreas was associated with an increased percentage of time (140 additional minutes) in near normoglycaemia (3.9-10.0 mmol/L) over
24 hours (overall weighted mean difference 9.62\% (95\% confidence interval $7.54 \%$ to $11.7 \%$ ); $\mathrm{I}^{2}=78 \%$, $\tau^{2}=24.09,32$ studies). This effect was consistent both for trials using artificial pancreas overnight $(7.16 \%$ (5.73\% to $8.58 \%) ; 0 \%, 0.0$, seven studies) or over 24 hours $(10.79 \%$ (7.88\% to $13.7 \%) ; 81 \%, 39.21,25$ studies; fig 2). The confidence interval for the overall effect estimate after applying the Hartung Knapp correction was $7.83 \%$ to $12.41 \%$, whereas the $95 \%$ prediction interval was $-0.63 \%$ to $19.87 \%$. Of note, the $95 \%$ prediction interval was above zero when the artificial pancreas was used overnight $(5.29 \%$ to $9.02 \%$ ), suggesting that artificial pancreas use will be beneficial in at least $95 \%$ of the individual study settings when applied overnight. However, the prediction interval contained negative values when applied over 24 hours $(-2.52 \%$ to $24.1 \%)$, and therefore might not be beneficial in some settings.

The favourable effect of artificial pancreas use over control treatment was more evident on the proportion of time in near normoglycaemia overnight (overall weighted mean difference $15.15 \%$ (95\% confidence interval $12.21 \%$ to $18.09 \%$ ); $\mathrm{I}^{2}=73 \%, \tau^{2}=43.48,31$ studies). This effect was consistent when artificial pancreas was used either only overnight (14.25\% (11.13\% to $17.37 \%) ; 63 \%, 19.39,14$ studies) or over 24 hours (16.44\% (10.88\% to $22.01 \%) ; 78 \%$, 99.63, 17 studies; fig 3), even when the Hartung Knapp correction was applied (appendix 13). Respective 95\% prediction intervals suggested that effect on time in near normoglycaemia overnight would be beneficial in at least $95 \%$ of the individual study settings when artificial pancreas was applied overnight $(4.04 \%$ to $24.45 \%)$, but not when applied over 24 hours $(-5.68 \%$ to $38.56 \%)$.

\section{Secondary outcmes}

Use of artificial pancreas had a favourable effect on time in hyperglycaemia (glucose concentrations $>10$ $\mathrm{mmol} / \mathrm{L}$ ) during the entire day. Compared with control treatment, this period was shortened by about two hours (overall weighted mean difference $-8.52 \%$ (95\% confidence interval $-11.14 \%$ to $-5.9 \%$ ); $\mathrm{I}^{2}=80 \%$, $\tau^{2}=28.98,22$ studies), both in trials using artificial pancreas overnight $(-6.0 \%(-8.4 \%$ to $-3.6 \%) ; 0 \%$, 0.0 , three studies) and those using artificial pancreas over 24 hours $(-9.08 \%(-12.23 \%$ to $-5.93 \%)$; $83 \%$, 37.53, 19 studies; fig 4). Similarly, the time when glucose concentrations were higher than $10.0 \mathrm{mmol} / \mathrm{L}$ overnight was also shortened compared with control treatment $(-11.12 \%(-13.8 \%$ to $-8.44 \%) ; 71 \%$, 26.13, 23 studies), both in trials that used artificial pancreas either only overnight $(-9.23 \%(-11.67 \%$ to $-6.79 \%) ; 51 \%, 8.26,12$ studies) or over 24 hours $(-13.86 \%(-19.83 \%$ to $-7.9 \%) ; 80 \%, 77.07,11$ studies; appendix 8).

Time when glucose concentrations were lower than $3.9 \mathrm{mmol} / \mathrm{L}$ over a 24 hour period was shortened with artificial pancreas use by about 20 minutes (overall weighted mean difference $-1.49 \%$ (95\% confidence interval $-1.86 \%$ to $-1.11 \%) ; \mathrm{I}^{2}=74 \%, \tau^{2}=0.59,29$ 


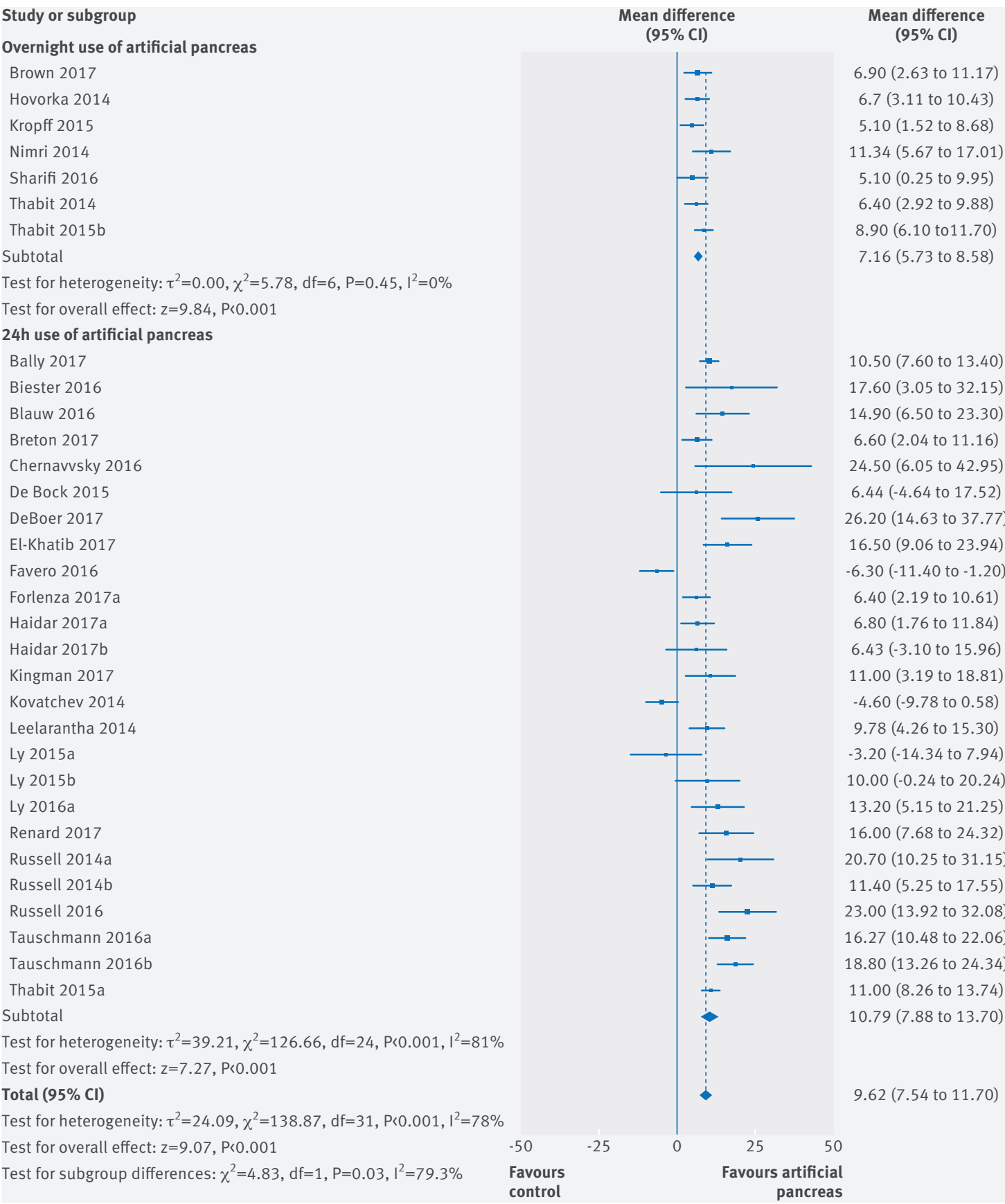

Fig 2 | Weighted mean difference in proportion (\%) of 24 hour period in near normoglycaemic range (glucose concentration 3.9-10.0 mmol/L), artificial pancreas use versus control treatment

studies), compared with control treatment (fig 5). Results were consistent for overnight time when concentrations were lower than $3.9 \mathrm{mmol} / \mathrm{L}(-2.22 \%$ $(-2.78 \%$ to $-1.65 \%) ; 72 \%, 1.34,29$ studies; appendix 9). Data on incidence of severe hypoglycaemia (that is, hypoglycaemia requiring third party assistance) were available in 27 studies (804 patients). Overall, incidence of severe hypoglycaemia was very low both in groups using artificial pancreas (six episodes) and control treatment (three episodes). Use of artificial pancreas was also associated with a reduction in overnight low blood glucose index $(-0.37$ ( -0.56 to -0.18); 85\%, 0.06, 11 studies).
Compared with control treatment, use of artificial pancreas had a favourable effect on mean levels of sensor blood glucose over 24 hours, which fell by $0.48 \mathrm{mmol} / \mathrm{L}$ (95\% confidence interval 0.3 to 0.66 ; $\mathrm{I}^{2}=84 \%, \tau^{2}=0.18,32$ studies; fig 6). Results were more favourable for mean levels of sensor blood glucose overnight (overall weighted mean difference $-0.81 \mathrm{mmol} / \mathrm{L}$ (-1.03 to -0.6); 78\%, 0.3, 35 studies; appendix 10). These findings were consistent with the effect of artificial pancreas use on $\mathrm{HbA}_{1 \mathrm{c}}(-0.26 \%$ $(-0.38 \%$ to $-0.13 \%)$; 0\%, 0.0, three studies; fig 7$)$. Finally, no difference between artificial pancreas use and control treatment was seen in the mean daily 


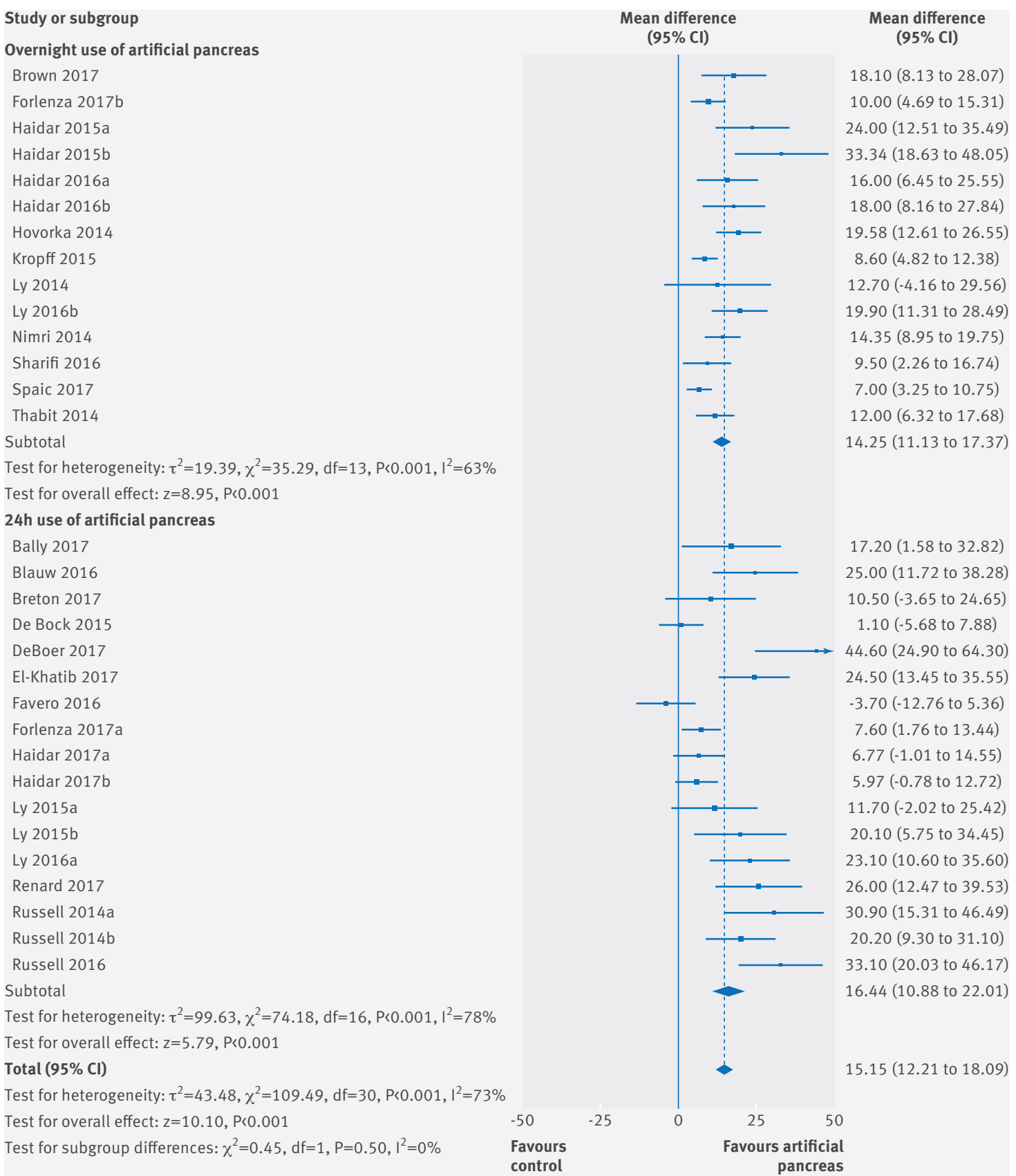

Fig 3 | Weighted mean difference in proportion (\%) of overnight period in near normoglycaemic range (glucose concentration 3.9-10.0 mmol/L), artificial pancreas use versus control treatment

needs for insulin (-0.21 IU (-1.64 to 1.22); 77\%, 4.45, 14 studies; appendix 11). Appendix 12 presents 95\% Hartung Knapp confidence intervals and prediction intervals for all outcomes.

\section{Sensitivity and subgroup analyses}

Results for the proportion of time in near normoglycaemia were similar in a sensitivity analysis including only trials at low risk of bias, both over 24 hours (overall weighted mean difference 11.64\% (95\% confidence interval $9.1 \%$ to $14.18 \%$ ); 10 studies) and overnight (20.18\% (13.18\% to $27.19 \%)$; five studies; fig 8 and fig 9). Similarly, results for near normoglycaemia did not differ in a series of sensitivity analyses excluding trials that using artificial pancreas in diabetes camps or including only trials using artificial pancreas in unsupervised patients in normal living conditions. This similarity was seen both for the 24 hour period (10.42\% (95\% confidence interval $8.63 \%$ to $12.2 \%$ ) and $10.67 \%$ (8.33\% to $13.01 \%$ ), respectively; appendices 13 and 14) and overnight period (13.47\% (10.41\% to $16.54 \%$ ) and $15.53 \%(10.12 \%$ to $20.94 \%)$, respectively; appendices 15 and 16).

We also did a post hoc sensitivity analysis excluding trials comparing artificial pancreas systems with low glucose suspend systems, to explore their effect on hypoglycaemia. Time when concentrations were lower than $3.9 \mathrm{mmol} / \mathrm{L}$ was shortened compared with control 


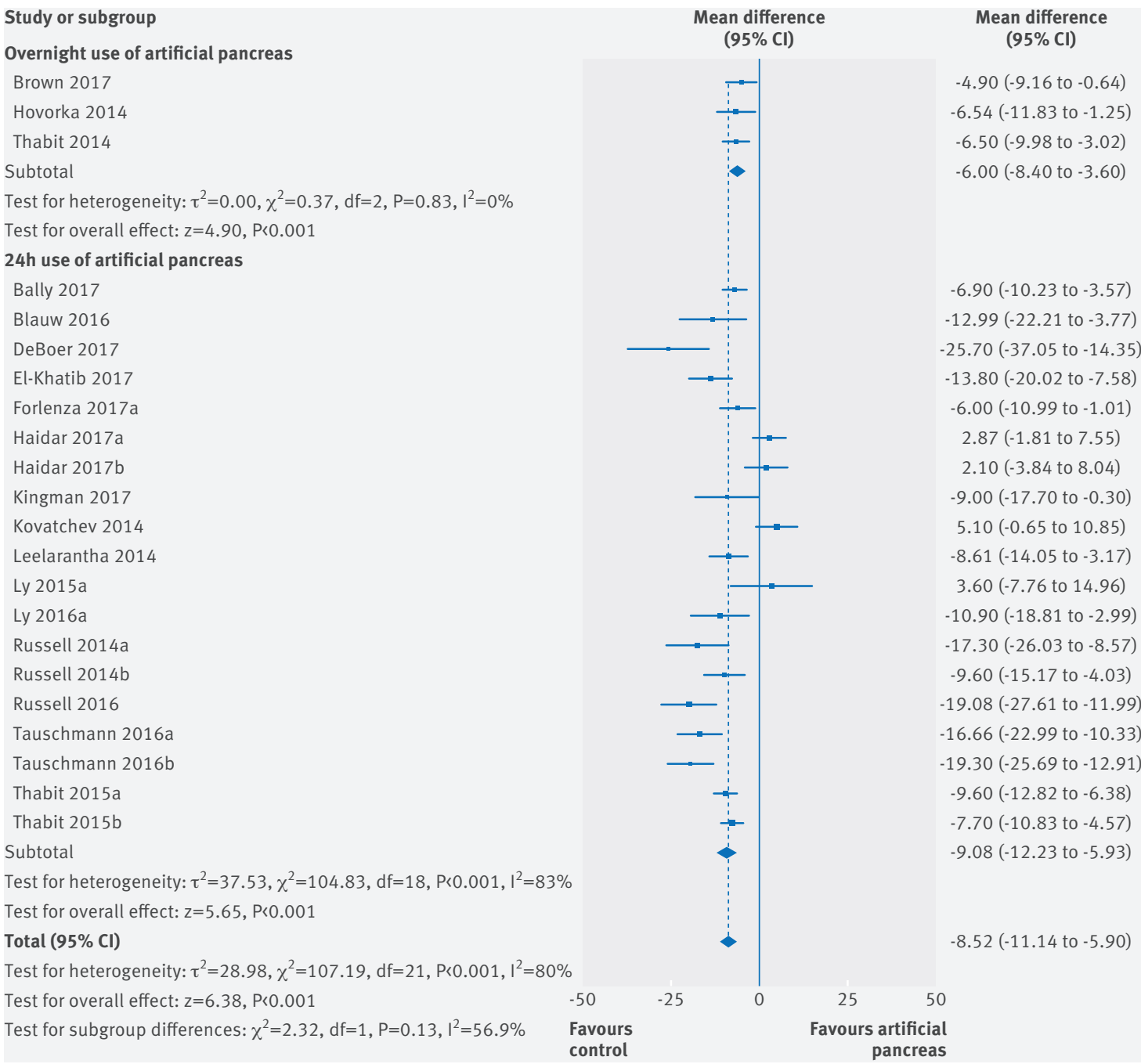

Fig 4 | Weighted mean difference in proportion (\%) of 24 hour period in hyperglycaemia (glucose concentration $>10.0$ $\mathrm{mmol} / \mathrm{L}$ ), artificial pancreas use versus control treatment

treatment (overall weighted mean difference $-1.59 \%$ (95\% confidence interval $-1.99 \%$ to $-1.19 \%$ ) for 24 hour period, $-2.53 \%(-3.18 \%$ to $-1.87 \%)$ for overnight period; appendices 17 and 18). Finally, for all outcomes, results were consistent with those of the main analysis in a prespecified subgroup analysis based on type of artificial pancreas used (that is, single hormone versus dual hormone artificial pancreas; table 2).

\section{Small study effects}

Both visually and formally, no evidence of small study effects was seen for the proportion of time in near normoglycaemia over 24 hours $(\mathrm{P}=0.129)$. However, evidence of small study effects was seen $(\mathrm{P}<0.001)$ for the proportion of time in near normoglycaemia overnight, and visual inspection of the contour enhanced funnel plot suggested that small negative studies were missing (appendix 19). Nevertheless, the adjusted meta-analytical estimate after use of the trim and fill method remained in favour of artificial pancreas use (weighted mean difference 10.39\% (95\% confidence interval $7.30 \%$ to $13.49 \%$ ), $\mathrm{P}<0.001)$.

\section{Discussion}

Key findings

Our data suggest that use of artificial pancreas is associated with almost two and a half additional hours in near normoglycaemia over a 24 hour period compared with control treatment, mainly due to its favourable effect during the overnight period. This finding was also verified by its effect on time in hyperglycaemia (two hours less than control treatment) and in hypoglycaemia (20 minutes less). Results were robust both for single and dual hormone systems, and were consistent in all sensitivity analyses performed-including an analysis restricted to trials under normal living conditions without remote monitoring, supporting the convenience and ease of use of artificial pancreas systems.

Finally, this favourable effect was also evident in the relative reduction of mean blood glucose levels by 0.48 $\mathrm{mmol} / \mathrm{L}$, which is consistent with the $\mathrm{HbA}_{1 \mathrm{c}}$ reduction of about $0.3 \%$ recorded in trials with a duration of more than eight weeks per intervention. ${ }^{446364}$ Overall, our results reflect the progress made over recent decades of extensive research and development in artificial pancreas use. 


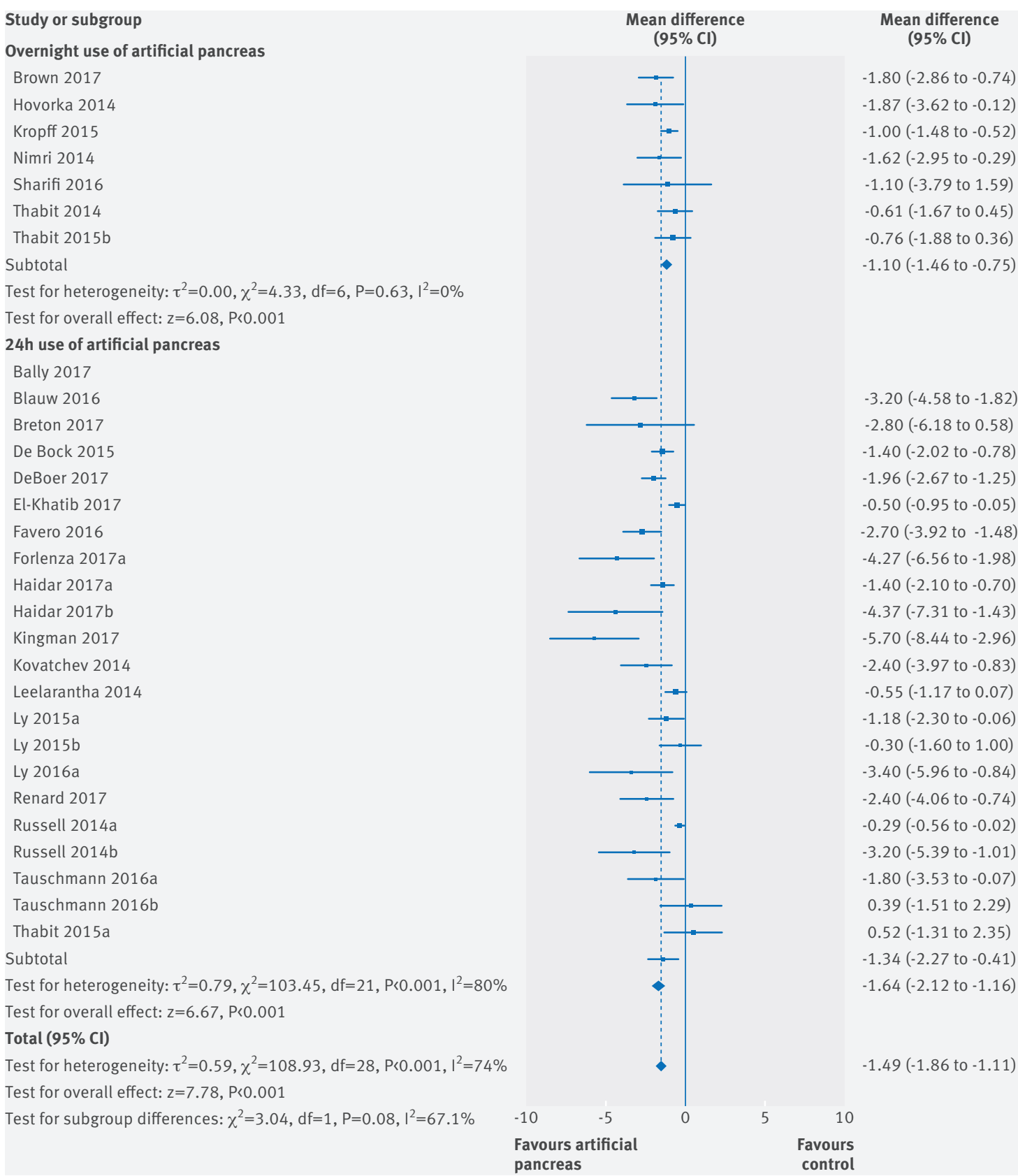

Fig 5 | Weighted mean difference in proportion (\%) of 24 hour period with glucose concentrations lower than 3.9 $\mathrm{mmol} / \mathrm{L}$, artificial pancreas use versus control treatment

\section{Comparison with other studies}

Despite heterogeneity in interventions and comparators used, our systematic review provides a valid and up to date overview on the use of artificial pancreas. An early pooled analysis of randomised controlled trials with artificial pancreas systems, published in 2011, included only four studies in an inpatient setting. ${ }^{8}$ The effect of artificial pancreas in the outpatient setting was examined in a recent systematic review and meta-analysis of 24 randomised controlled trials (585 participants). ${ }^{10}$ However, validity and clinical interpretation potential of the results were undermined by methodological decisions regarding the definition of outcomes, handling of median values, and exclusion of evidence from grey literature sources, potentially missing a substantial amount of evidence. ${ }^{65}$ Our systematic review and meta-analysis incorporated a much larger pool of eligible studies $(n=41)$ and participants $(n=1042)$ and assessed a broader variety of outcomes, focusing on outcome definitions considered most important in trials evaluating artificial pancreas systems. ${ }^{66-68}$

Furthermore, Weisman and colleagues analysed only 24 hour outcomes for studies investigating artificial pancreas use for 24 hour periods and analysed only overnight outcomes for studies investigating artificial pancreas use overnight, even when individual trials provided data for both periods. ${ }^{10}$ Instead, our systematic review dealt with the research question 


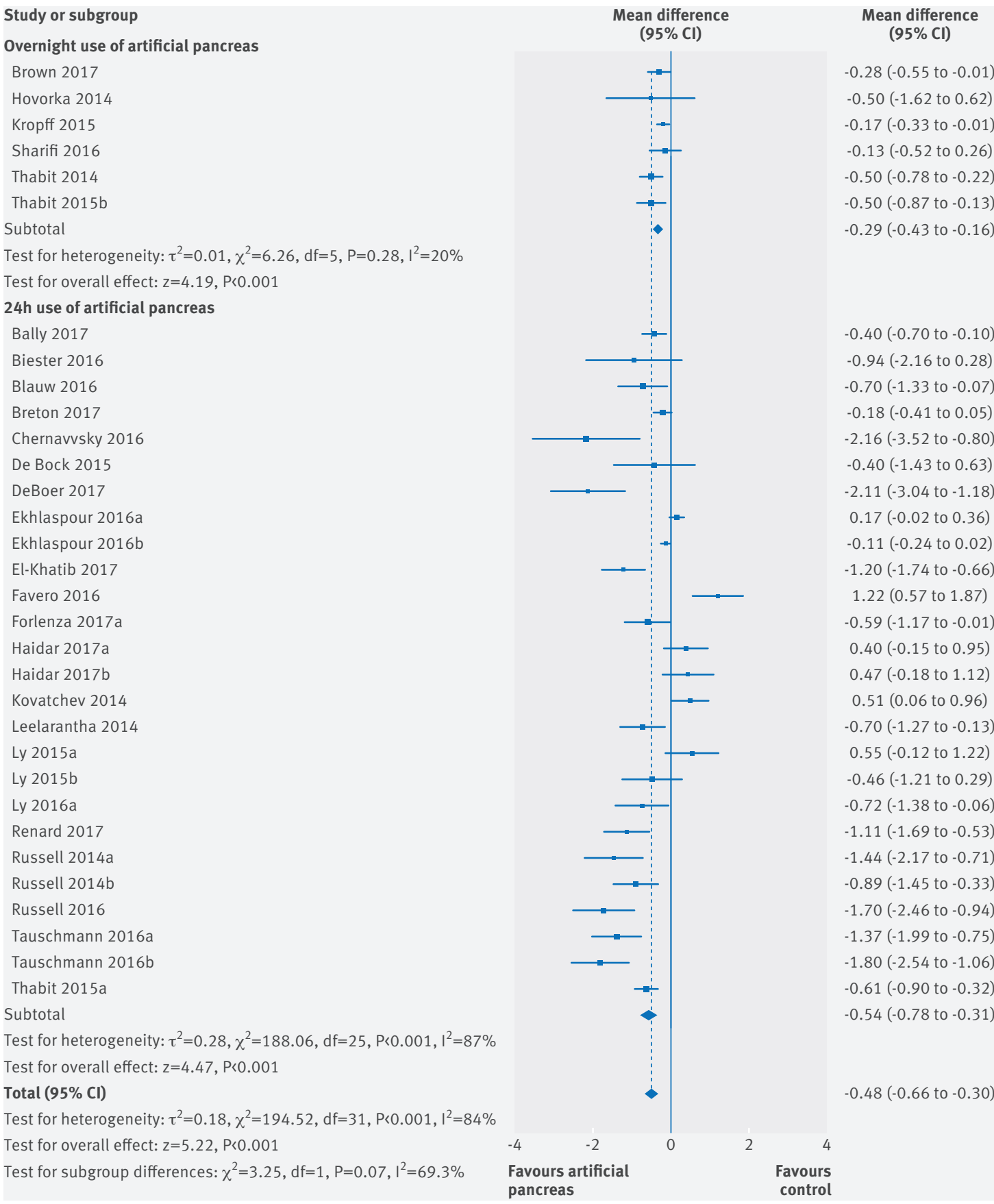

Fig 6 | Weighted mean difference in mean levels of sensor blood glucose (mmol/L) over 24 hours, artificial pancreas use versus control treatment

Study or subgroup

Kropff 2015

Thabit 2014

Thabit 2015b

Total $(95 \% \mathrm{Cl})$

Test for heterogeneity: $\tau^{2}=0.00, \chi^{2}=0.60, d f=2, P=0.74, I^{2}=0 \%$

Test for overall effect: $z=4.04, P<0.001$

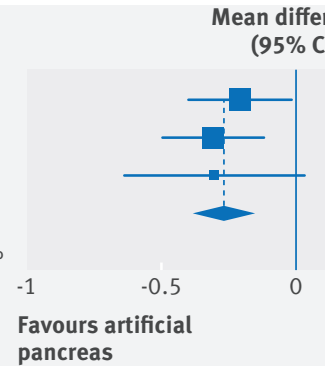

Fig 7 | Weighted mean difference in change in $\mathrm{HbA}_{1 \mathrm{c}}(\%)$, artificial pancreas use versus control treatment 


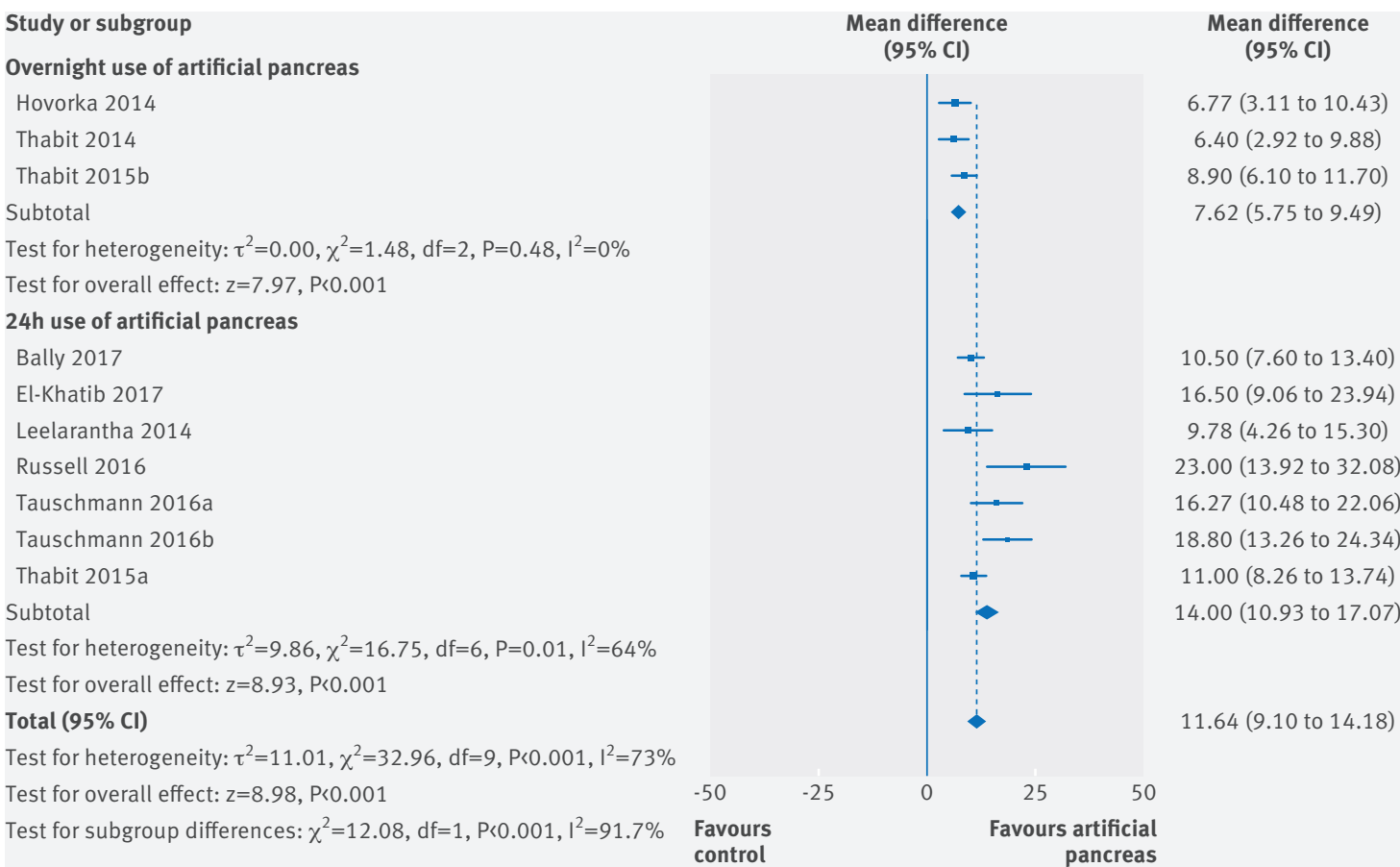

Fig 8 | Weighted mean difference in proportion (\%) of 24 hour period in near normoglycaemic range (glucose concentration 3.9-10.0 mmol/L), artificial pancreas use versus control treatment. Sensitivity analysis includes only trials at low risk of bias

by conducting separate analyses based on all four combinations of outcome assessment period ( 24 hours or overnight) and duration of intervention use (for 24 hours or solely overnight).

\section{Strengths and limitations of study}

Composition of the review team ensured appropriate methodological and field expertise, but also access to additional study data from individual studies. ${ }^{41} 45$ 60-63 To ensure internal validity of our conclusions, we implemented current guidelines for the conduct and reporting of systematic reviews, ${ }^{12}$ and adhered to a prespecified protocol with minimal deviations. We undertook a comprehensive search of multiple databases without imposing any restrictions based on language or publication type, and assessed quality of trials using valid methodological tools. Moreover, we synthesised existing data using appropriate methodology to account for inappropriate reporting and analysis methods used in some of the trials

\section{Study or subgroup}

Overnight use of artificial pancreas

Hovorka 2014

Thabit 2014

Subtotal

Test for heterogeneity: $\tau^{2}=18.21, \chi^{2}=2.73, \mathrm{df}=1, \mathrm{P}=0.10, \mathrm{I}^{2}=63 \%$

Test for overall effect: $z=4.10, P<0.001$

$24 \mathrm{~h}$ use of artificial pancreas

Bally 2017

El-Khatib 2017

Russell 2016

Subtotal

Test for heterogeneity: $\tau^{2}=9.27, \chi^{2}=2.41, \mathrm{df}=2, \mathrm{P}=0.30, \mathrm{I}^{2}=17 \%$

Test for overall effect: $z=6.09, P<0.001$

Total $(95 \% \mathrm{Cl})$

Test for heterogeneity: $\tau^{2}=37.49, \chi^{2}=10.94, d f=4, P=0.03, I^{2}=63 \%$

Test for overall effect: $z=5.65, P<0.001$

Test for subgroup differences: $\chi^{2}=3.16, \mathrm{df}=1, \mathrm{P}=0.08, \mathrm{I}^{2}=68.4 \%$ Favours

Favours
control

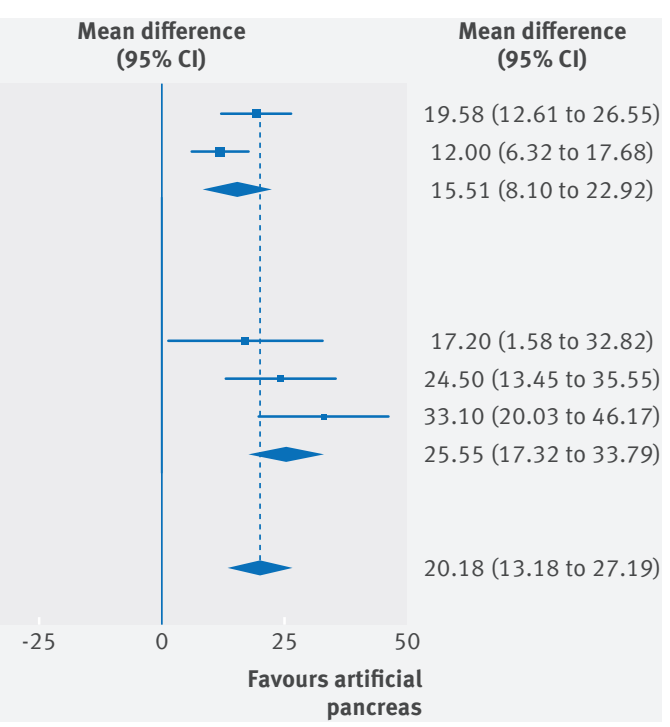

ean differen

19.58 (12.61 to 26.55$)$

12.00 (6.32 to 17.68$)$

15.51 (8.10 to 22.92$)$

7.20 (1.58 to 32.82$)$

$24.50(13.45$ to 35.55$)$

$33.10(20.03$ to 46.17$)$

25.55 (17.32 to 33.79$)$

Fig 9 | Weighted mean difference in proportion (\%) of overnight period in near normoglycaemic range (glucose concentration 3.9-10.0 mmol/L), artificial pancreas use versus control treatment. Sensitivity analysis includes only trials at low risk of bias 


\begin{tabular}{|c|c|c|c|}
\hline \multirow[b]{2}{*}{ Outcome and time period } & \multirow[b]{2}{*}{ No of studies (single/dual hormone) } & \multicolumn{2}{|c|}{ Artificial pancreas system v control treatment* (weighted mean difference $\left.\left(95 \% C^{\prime}\right), I^{2}, \tau^{2}\right)$} \\
\hline & & Single hormone & Dual hormone \\
\hline \multicolumn{4}{|c|}{ Proportion (\%) of time in near normoglycaemia (glucose concentration 3.9-10.0 mmol/L) } \\
\hline $24 \mathrm{~h}$ & $26 / 6$ & 8.53 (6.34 to 10.72$), 78 \%, 22.00$ & $15.16(10.68$ to 19.63$), 43 \%, 13.08$ \\
\hline Overnight & $23 / 8$ & 12.77 (9.82 to 15.71$), 68 \%, 29.73$ & 2.84 (15.08 to 30.60$), 74 \%, 88.82$ \\
\hline \multicolumn{4}{|c|}{ Proportion (\%) of time with glucose concentration greater than $>10.0 \mathrm{mmol} / \mathrm{L}$} \\
\hline $24 \mathrm{~h}$ & $16 / 6$ & $-7.52(-10.38$ to -4.66$), 80 \%, 24.96$ & -11.58 (-18.17 to -4.99$), 81 \%, 36.43$ \\
\hline Overnight & $15 / 8$ & $-8.4(-10.22$ to -6.58$), 24 \%, 2.82$ & $-17.21(-25.58$ to -8.85$), 87 \%, 121.35$ \\
\hline \multicolumn{4}{|c|}{ Proportion (\%) of time with glucose concentration lower than $3.9 \mathrm{mmol} / \mathrm{L}$} \\
\hline $24 \mathrm{~h}$ & $24 / 5$ & $-1.28(-1.65$ to -0.92$), 72 \%, 0.45$ & $-2.95(-4.03$ to -1.87$), 30 \%, 0.45$ \\
\hline Overnight & $24 / 7$ & $-1.82(-2.38$ to -1.27$), 70 \%, 1.00$ & $-4.04(-5.59$ to -2.48$), 47 \%, 1.93$ \\
\hline Low blood glucose index, overnight & $11 / 0$ & $-0.37(-0.56$ to -0.18$), 85 \%, 0.06$ & Not estimable \\
\hline \multicolumn{4}{|l|}{ Mean sensor glucose value (mmol/L) } \\
\hline $24 \mathrm{~h}$ & $25 / 7$ & $-0.41(-0.61$ to -0.20$), 83 \%, 0.19$ & $-0.76(-1.31$ to -0.22$), 89 \%, 0.45$ \\
\hline Overnight & $29 / 8$ & $-0.67(-0.89$ to -0.45$), 76 \%, 0.24$ & $-1.47(-2.14$ to -0.79$), 80 \%, 0.72$ \\
\hline Daily insulin need (IU), over $24 \mathrm{~h}$ & $13 / 1$ & $-0.47(-1.84$ to 0.89$), 76 \%, 3.78$ & Not estimable \\
\hline
\end{tabular}

included. In addition, we conducted a range of sensitivity analyses excluding trials using remote monitoring or trials at high risk of bias, to examine clinical relevance and robustness of our findings.

We acknowledge several limitations at the evidence and review level. Most trials had a small sample size, limiting the precision of our effect estimates. Despite using broad inclusion criteria, existing studies provided limited insight regarding clinically relevant subgroups, such as those with increased hypoglycaemia burden, hypoglycaemia unawareness, gastroparesis, blindness, high $\mathrm{HbA}_{1 c}$, treated with corticosteroids, or from ethnic minorities. ${ }^{69}$ Many trials were at high or unclear risk of bias owing to suboptimal reporting. Specifically, most trials reported effect estimates for outcomes related to hypoglycaemia using median values and interquartile ranges, thus we had to impute mean and standard deviation values for the meta-analyses. In addition, several crossover trials reported results as parallel group studies, ${ }^{47-49}$ which also required use of imputation methods to allow synthesis of results.

Furthermore, we did not register our protocol at a publicly available database, and submitted it only for internal peer review. We focused on surrogate outcomes and did not extract evidence for specific patient outcomes such as quality of life, incidence of ketoacidosis, or catheter occlusion. Instead, we adopted a practical approach focusing on outcomes that we expected to be most and best reported in trials. ${ }^{68}$ Moreover, for missing or inappropriately reported data, we refrained from contacting study authors other than those who were members of the review group, but used appropriate methodology to impute data. $^{70}$

Finally, most analyses had a high degree of heterogeneity, which could be attributed to differences in continuous glucose monitoring used, sensor accuracy and performance, compliance with artificial pancreas use in supervised and unsupervised settings, and comparators used during control treatment if sensor glucose readings were or were not available.
These differences could explain wide prediction intervals that included zero values for most outcomes in trials using artificial pancreas over 24 hours; thus, related findings should be interpreted with caution. By contrast, strong evidence indicated that overnight use of artificial pancreas would be beneficial for outcomes regarding time in near normoglycaemia, hyperglycaemia, or hypoglycaemia (95\% prediction intervals excluding zero values), suggesting that this treatment effect can be expected in future patients.

\section{Implications}

Our study highlights some pitfalls in the conduct and reporting of artificial pancreas trials. Many trials had a short duration or were designed to assess the feasibility or safety, rather than long term effectiveness. Despite existing guidance, we noted significant variation in outcomes assessed and metrics used..$^{71}$ Research groups should report a minimum set of agreed outcome measures and respective metrics. ${ }^{66-68}$ To ensure the clinical relevance and feasibility of this core outcome set, it is crucial that its development involves all key stakeholders, including patients, their families, clinicians, researchers, statisticians, methodologists, industry representatives, regulatory authorities, and funders.

To maximise the yield of information and to facilitate analysis and synthesis of evidence overall, the use of a common repository for data on individual patients could be agreed on. ${ }^{72}$ Such repositories would facilitate free dissemination of raw trial data, allowing for replication of previous research findings using various analysis approaches (for example, a repeated measures analysis) of clinically relevant outcomes. Moreover, to enhance the external validity of evidence, future trials should broaden inclusion criteria and recruit more heterogeneous populations, including ethnic minorities.

The performance of current artificial pancreas systems could be enhanced by the optimisation of system components. Use of novel insulin analogues with faster pharmacokinetics, ${ }^{74}$ the development of 
glucagon preparation stable at room temperature, and integration of artificial pancreas components into one device could further enhance user experience and artificial pancreas usefulness, and thus increase uptake. Future research should explore the potential differences between individual components (algorithms, continuous glucose monitoring) and determine their clinical relevance.

Upcoming trials should clarify the differences between single hormone and dual hormone systems, and explore artificial pancreas use in relevant groups of people with type 2 diabetes such as those with inpatient hyperglycaemia. ${ }^{75}$ Moreover, the effect of artificial pancreas use on quality of life and on reducing patient burden should be further explored, ${ }^{76}$ considering that patients with type 1 diabetes and their carers have shown a positive attitude towards artificial pancreas systems. ${ }^{77-79}$ Finally, to support adoption, cost effectiveness should be assessed to allow for reimbursement by various healthcare systems, and ensure that adequate infrastructure exists.

\section{Conclusions}

Our systematic review and meta-analysis has shown that artificial pancreas systems are an efficacious and safe treatment approach for people with type 1 diabetes, leading to increased time in near normoglycaemic range, and reduced time in hypoglycaemia and hyperglycaemia. The results were verified for all types of artificial pancreas and in all sensitivity analyses. Further research with rigorous studies, cooperation of research groups in terms of outcome reporting, and cost effectiveness data are required to verify these findings and support adoption of artificial pancreas systems in clinical practice.

Contributors: $\mathrm{EB}, \mathrm{HT}$, and AT conceived and designed the study. $E B$ and EA did the scientific literature search. EB, KK, EA, and AT did literature screening. EB, EA, TK, and AT extracted data. EB, EA, and AT did quality assessment of the included studies. EB, TK, A-BH, RH, and AT did the analyses. EB, KK, HT, MT, TK, RH, and AT wrote the first draft of the report. All authors contributed to interpretation and edited the draft report. AT is the study guarantor, had full access to all the trial level data in the study, takes responsibility for the integrity of the data, and accuracy of the data analysis, and had the final responsibility to submit for publication.

Funding: The study was partly funded by the Aristotle University Research Committee (ELKE AUTh), and supported by the National Institute for Health Research Cambridge Biomedical Research Centre and Wellcome Strategic Award $(100574 / Z / 12 / Z)$. The funder of the study had no role in study design, data collection, data analysis, data interpretation, or writing of the report.

Competing interests: All authors have completed the ICMJE uniform disclosure at www.icmje.org/coi_disclosure.pdf and declare: support from the Aristotle University Research Committee, National Institute for Health Research Cambridge Biomedical Research Centre, and Wellcome Strategic Award for the submitted work; KK reports honorarium fees from Medtronic, Novo Nordisk, and Sanofi outside the submitted work; MT reports personal fees from Medtronic and Novo Nordisk outside the submitted work; RH reports personal fees from Eli Lilly, Novo Nordisk, BBraun, and Medtronic, grants from the National Institute for Health Research Cambridge Biomedical Research Centre, and Wellcome Strategic Award outside the submitted work, and reports patents and patent applications; AT reports honorarium fees from AstraZeneca, Boehringer Ingelheim, and Novo Nordisk outside the submitted work; no other relationships or activities that could appear to have influenced the submitted work.

Ethical approval: Ethical approval not required.

Data sharing: No additional data available.
The lead author affirms that the manuscript is an honest, accurate, and transparent account of the study being reported; that no important aspects of the study have been omitted; and that any discrepancies from the study as planned have been explained.

This is an Open Access article distributed in accordance with the terms of the Creative Commons Attribution (CC BY 4.0) license, which permits others to distribute, remix, adapt and build upon this work, for commercial use, provided the original work is properly cited. See: http://creativecommons.org/licenses/by/4.0/.

1 National Institute for Health and Care Excellence. Continuous subcutaneous insulin infusion for the treatment of diabetes mellitus. NICE Technology Appraisal Guidance 151. 2008 [8 July 2014]. www. nice.org.uk/Guidance/TA151.

2 Yeh HC, Brown TT, Maruthur N, et al. Comparative effectiveness and safety of methods of insulin delivery and glucose monitoring for diabetes mellitus: a systematic review and meta-analysis. Ann Intern Med 2012;157:336-47. doi:10.7326/0003-4819-157-5201209040-00508

3 Group RS, REPOSE Study Group. Relative effectiveness of insulin pump treatment over multiple daily injections and structured education during flexible intensive insulin treatment for type 1 diabetes: cluster randomised trial (REPOSE). BMJ 2017;356:11285.

4 Riemsma R, Corro Ramos I, Birnie R, et al. Integrated sensoraugmented pump therapy systems [the MiniMed Paradigm Veo system and the Vibe and G4 PLATINUM CGM (continuous glucose monitoring) system] for managing blood glucose levels in type 1 diabetes: a systematic review and economic evaluation. Health Technol Assess 2016;20:1-251. doi:10.3310/hta20170

5 Bergenstal RM, Tamborlane WV, Ahmann A, et al, STAR 3 Study Group. Effectiveness of sensor-augmented insulin-pump therapy in type 1 diabetes. N Engl J Med 2010;363:311-20. doi:10.1056/ NEJMoa1002853

6 Agrawal P, Welsh JB, Kannard B, Askari S, Yang Q, Kaufman FR. Usage and effectiveness of the low glucose suspend feature of the Medtronic Paradigm Veo insulin pump. J Diabetes Sci Technol 2011;5:1137-41. doi:10.1177/193229681100500514

Roze S, Smith-Palmer J, Valentine WJ, et al. Long-term health economic benefits of sensor-augmented pump therapy vs continuous subcutaneous insulin infusion alone in type 1 diabetes: a U.K. perspective. J Med Econ 2016;19:236-42. doi:10.3111/13696998. 2015.1113979

8 Kumareswaran K, Elleri D, Allen JM, et al. Meta-analysis of overnight closed-loop randomized studies in children and adults with type 1 diabetes: the Cambridge cohort. / Diabetes Sci Technol 2011;5:1352-62. doi:10.1177/193229681100500606

9 Battelino T, Omladič JS, Phillip M. Closed loop insulin delivery in diabetes. Best Pract Res Clin Endocrinol Metab 2015;29:315-25. doi:10.1016/j.beem.2015.03.001

10 Weisman A, Bai JW, Cardinez M, Kramer CK, Perkins BA. Effect of artificial pancreas systems on glycaemic control in patients with type 1 diabetes: a systematic review and meta-analysis of outpatient randomised controlled trials. Lancet Diabetes Endocrinol 2017;5: 501-12. doi:10.1016/S2213-8587(17)30167-5

11 Bergenstal RM, Garg S, Weinzimer SA, et al. Safety of a hybrid closed-loop insulin delivery system in patients with type 1 diabetes. JAMA 2016;316:1407-8. doi:10.1001/jama.2016.11708

12 Liberati A, Altman DG, TetzlaffJ, et al. The PRISMA statement for reporting systematic reviews and meta-analyses of studies that evaluate health care interventions: explanation and elaboration. Ann Intern Med 2009;151:W65-94. doi:10.7326/0003-4819-151-4200908180-00136

13 The Cochrane Collaboration. Higgins JPT, Green S, eds. Cochrane Handbook for Systematic Reviews of Interventions version 5.1.0 [updated March 2011]. 2011. http://handbook.cochrane.org.

14 Centre for Reviews and Dissemination. Systematic reviews: CRD's guidance for undertaking reviews in health care York: University of York; 2009 [12/8/2016]. https://www.york.ac.uk/crd/SysRev/!SSL!/ WebHelp/SysRev3.htm

15 Kovatchev BP, Cox DJ, Gonder-Frederick LA, Young-Hyman D, Schlundt D, Clarke W. Assessment of risk for severe hypoglycemia among adults with IDDM: validation of the low blood glucose index. Diabetes Care 1998;21:1870-5. doi:10.2337/diacare.21.11.1870

16 DerSimonian R, Laird N. Meta-analysis in clinical trials. Control Clin Trials 1986;7:177-88. doi:10.1016/0197-2456(86)90046-2

17 Riley RD, Higgins JP, Deeks JJ. Interpretation of random effects metaanalyses. BMJ 2011;342:d549. doi:10.1136/bmj.d549

18 IntHout J, Ioannidis JP, Borm GF. The Hartung-Knapp-Sidik-Jonkman method for random effects meta-analysis is straightforward and considerably outperforms the standard DerSimonian-Laird method. BMC Med Res Methodol 2014;14:25. doi:10.1186/1471-2288-14-25

19 Wan X, Wang W, Liu J, Tong T. Estimating the sample mean and standard deviation from the sample size, median, range and/or interquartile range. BMC Med Res Methodol 2014;14:135. doi:10.1186/1471-2288-14-135 
20 Elbourne DR, Altman DG, Higgins JP, Curtin F, Worthington HV, Vail A. Meta-analyses involving cross-over trials: methodological issues. Int J Epidemiol 2002;31:140-9. doi:10.1093/ije/31.1.140

21 Ding H, Hu GL, Zheng XY, Chen Q, Threapleton DE, Zhou ZH. The method quality of cross-over studies involved in Cochrane Systematic Reviews. PLoS One 2015;10:e0120519. doi:10.1371/journal. pone.0120519

22 Egger M, Davey Smith G, Schneider M, Minder C. Bias in metaanalysis detected by a simple, graphical test. BMJ 1997:315:629-34. doi:10.1136/bmj.315.7109.629

23 Peters JL, Sutton AJ, Jones DR, Abrams KR, Rushton L. Contourenhanced meta-analysis funnel plots help distinguish publication bias from other causes of asymmetry. / Clin Epidemiol 2008;61: 991-6. doi:10.1016/j.jclinepi.2007.11.010

24 Peters JL, Sutton AJ, Jones DR, Abrams KR, Rushton L. Performance of the trim and fill method in the presence of publication bias and between-study heterogeneity. Stat Med 2007;26:4544-62. doi:10.1002/sim.2889

25 Bally L, Thabit H, Kojzar H, et al. Day-and-night glycaemic control with closed-loop insulin delivery versus conventional insulin pump therapy in free-living adults with well controlled type 1 diabetes: an open-label, randomised, crossover study. Lancet Diabetes Endocrinol 2017:5:261-70. doi:10.1016/S22138587(17)30001-3

26 Biester T, Muller I, Remus K, et al. 60 hours hybrid-closed-loop ( $\mathrm{HCL}$ ) in everyday life. The DREAM5-study. Pediatr Diabetes 2016:17:146.

27 Blauw H, van Bon AC, Koops R, DeVries JH, on behalf of the PCDIAB consortium. Performance and safety of an integrated bihormonal artificial pancreas for fully automated glucose control at home. Diabetes Obes Metab 2016;18:671-7. doi:10.1111/dom.12663

28 Breton MD, Cherñavvsky DR, Forlenza GP, et al. Closed-loop control during intense prolonged outdoor exercise in adolescents with type 1 diabetes: The artificial pancreas ski study. Diabetes Care 2017;40:1644-50. doi:10.2337/dc17-0883

29 Brown SA, Breton MD, Anderson SM, et al. Overnight closed-loop control improves glycemic control in a multicenter study of adults with type 1 diabetes. J Clin Endocrinol Metab 2017;102:3674-82. doi:10.1210/jc.2017-00556

30 Cherñavvsky DR, DeBoer MD, Keith-Hynes P, et al. Use of an artificial pancreas among adolescents for a missed snack bolus and an underestimated meal bolus. Pediatr Diabetes 2016;17:28-35. doi:10.1111/pedi.12230

31 de Bock MI, Roy A, Cooper MN, et al. Feasibility of outpatient 24 hour closed-loop insulin delivery. Diabetes Care 2015;38:e186-7. doi:10.2337/dc15-1047

32 DeBoer MD, Breton MD, Wakeman C, et al. Performance of an artificial pancreas system for young children with type 1 diabetes. Diabetes Technol Ther 2017;19:293-8. doi:10.1089/dia.2016.0424

33 Ekhlaspour L, Elkhatib F, Balliro C, et al. Outpatient glycemic management in type 1 diabetes with insulin-only vs. Bihormonal configurations of a bionic pancreas. Diabetes 2016 65(suppl 1):A21.

34 El-Khatib FH, Balliro C, Hillard MA, et al. Home use of a bihormonal bionic pancreas versus insulin pump therapy in adults with type 1 diabetes: a multicentre randomised crossover trial. Lancet, 2016.

35 Del Favero S, Boscari F, Messori M, et al. Randomized summer camp crossover trial in 5-to 9-year-old children: outpatient wearable artificial pancreas is feasible and safe. Diabetes Care 2016;39: 1180-5. doi:10.2337/dc15-2815

36 Forlenza GP, Raghinaru D, Cameron F, et al, In-Home ClosedLoop (IHCL) Study Group. Predictive hyperglycemia and hypoglycemia minimization: In-home double-blind randomized controlled evaluation in children and young adolescents. Pediatr Diabetes 2017. doi:10.1111/pedi.12603

37 Forlenza GP, Deshpande S, Ly TT, et al. Application of zone model predictive control artificial pancreas during extended use of infusion set and sensor: a randomized crossover-controlled home-use trial (correction in: Diabetes Care 2017;40:1096-102). Diabetes Care 2017:40:1096-102 doi:10.2337/dc17-0500

38 Haidar A, Legault L, Matteau-Pelletier L, et al. Outpatient overnight glucose control with dual-hormone artificial pancreas, single hormone artificial pancreas, or conventional insulin pump therapy in children and adolescents with type 1 diabetes: an open-label, randomised controlled trial. Lancet Diabetes Endocrinol 2015;3 595-604. doi:10.1016/S2213-8587(15)00141-2

39 Haidar A, Rabasa-Lhoret R, Legault L, et al. Single- and dual-hormone artificial pancreas for overnight glucose control in type 1 diabetes. J Clin Endocrinol Metab 2016;101:214-23. doi:10.1210/ jc. 2015-3003

40 Haidar A, Messier V, Legault L, Ladouceur M, Rabasa-Lhoret R. Outpatient 60-hour day-and-night glucose control with dualhormone artificial pancreas, single-hormone artificial pancreas, or sensor-augmented pump therapy in adults with type 1 diabetes: An open-label, randomised, crossover, controlled trial. Diabetes Obes Metab 2017;19:713-20. doi:10.1111/dom.12880
41 Hovorka R, Elleri D, Thabit $\mathrm{H}$, et al. Overnight closed-loop insulin delivery in young people with type 1 diabetes: a free-living, randomized clinical trial. Diabetes Care 2014:37:1204-11. doi:10.2337/dc13-2644

42 Kingman RS, Robic JL, Buckingham BA, Clinton P, Kovatchev BP, Anderson SM. Restoration of hypoglycemia awareness with closedloop therapy. Diabetes 2017;66(suppl 1):A94-5.

43 Kovatchev BP, Renard E, Cobelli C, et al. Safety of outpatient closedloop control: first randomized crossover trials of a wearable artificial pancreas. Diabetes Care 2014;37:1789-96. doi:10.2337/ dc13-2076

44 Kropff J, Del Favero S, Place J, et al, AP@home consortium. 2 month evening and night closed-loop glucose control in patients with type 1 diabetes under free-living conditions: a randomised crossover trial. Lancet Diabetes Endocrinol 2015;3:939-47. doi:10.1016/S22138587(15)00335-6

45 Leelarathna L, Dellweg S, Mader JK, et al, AP@home Consortium. Day and night home closed-loop insulin delivery in adults with type 1 diabetes: three-center randomized crossover study. Diabetes Care 2014;37:1931-7. doi:10.2337/dc13-2911

46 Ly TT, Breton MD, Keith-Hynes P, et al. Overnight glucose control with an automated, unified safety system in children and adolescents with type 1 diabetes at diabetes camp. Diabetes Care 2014;37:2310-6. doi:10.2337/dc14-0147

47 Ly TT, Chernavvsky D, DeSalvo D, Shanmugham S, Breton M, Buckingham B. Day and night closed-loop control with the DIAS system in patients with type 1 diabetes at camp. Diabetes Technol Ther 2015;17:A97

48 Ly TT, Roy A, Grosman B, et al. Day and night closed-loop control using the integrated Medtronic hybrid closed-loop system in type 1 diabetes at diabetes camp. Diabetes Care 2015;38:1205-11. doi:10.2337/dc14-3073

49 Ly TT, Buckingham BA, DeSalvo DJ, et al. Day-and-night closed-loop control using the unified safety system in adolescents with type 1 diabetes at camp. Diabetes Care 2016;39:e106-7. doi:10.2337/ dc16-0817

50 Ly TT, Keenan DB, Roy A, et al. Automated overnight closed-loop control using a proportional-integral-derivative algorithm with insulin feedback in children and adolescents with type 1 diabetes at diabetes camp. Diabetes Technol Ther 2016;18:377-84 doi:10.1089/dia.2015.0431

51 Nimri R, Muller I, Atlas E, et al. MD-Logic overnight control for 6 weeks of home use in patients with type 1 diabetes. randomized crossover trial. Diabetes Care 2014;37:3025-32. doi:10.2337/dc14-0835

52 Nimri R, Bratina N, Kordonouri O, et al. MD-Logic overnight type 1 diabetes control in home settings: multicenter, multinational, single blind, randomized trial. Diabetes Obes Metab 2017;19:553-61.

53 Phillip M, Battelino T, Atlas E, et al. Nocturnal glucose control with an artificial pancreas at a diabetes camp. N Engl / Med 2013;368: 824-33. doi:10.1056/NEIMoa1206881

54 Renard E, Tubiana-Rufi N, Bonnemaison-Gilbert E, et al. Closed-loop outperforms threshold-low-glucose suspend insulin delivery on glucose control in prepubertal outpatients with type 1 diabetes. Diabetes 2017;66(suppl 1):A79

55 Russell SJ, El-Khatib FH, Sinha M, et al. Outpatient glycemic control with a bionic pancreas in type 1 diabetes. $N$ Engl Med 2014;371:313-25. doi:10.1056/NEJMoa1314474

56 Russell SJ, Hillard MA, Balliro C, et al. Day and night glycaemic contro with a bionic pancreas versus conventional insulin pump therapy in preadolescent children with type 1 diabetes: a randomised crossover trial. Lancet Diabetes Endocrinol 2016;4:233-43. doi:10.1016/ S2213-8587(15)00489-1

57 Schierloh U, Wilinska M, Thabit H, et al. Validation of a closed loop system in paediatric patients, 6 to 12 years, with type 1 diabetes. Diabetes Technol Ther 2015;17:A98-9.

58 Sharifi A, De Bock MI, Jayawardene D, et al. Glycemia, treatment satisfaction, cognition, and sleep quality in adults and adolescents with type 1 diabetes when using a closed-loop system overnight versus sensor-augmented pump with low-glucose suspend function: a randomized crossover study. Diabetes Technol Ther 2016;18: 772-83. doi:10.1089/dia.2016.0288

59 Spaic T, Driscoll M, Raghinaru D, et al, In-Home Closed-Loop (IHCL) Study Group. Predictive hyperglycemia and hypoglycemia minimization: in-home evaluation of safety, feasibility, and efficacy in overnight glucose control in type 1 diabetes. Diabetes Care 2017:40:359-66. doi:10.2337/dc16-1794

60 Tauschmann M, Allen JM, Wilinska ME, et al. Home use of day-andnight hybrid closed-loop insulin delivery in suboptimally controlled adolescents with type 1 diabetes: a 3-week, free-living, randomized crossover trial. Diabetes Care 2016;39:2019-25. doi:10.2337/ dc16-1094

61 Tauschmann M, Allen JM, Wilinska ME, et al. Day-and-night hybrid closed-loop insulin delivery in adolescents with type 1 diabetes: a free-living, randomized clinical trial. Diabetes Care 2016;39: 1168-74. doi:10.2337/dc15-2078 
62 Thabit H, Lubina-Solomon A, Stadler M, et al. Home use of closedloop insulin delivery for overnight glucose control in adults with type 1 diabetes: a 4-week, multicentre, randomised crossover study. Lancet Diabetes Endocrinol 2014;2:701-9. doi:10.1016/S22138587(14)70114-7

63 Thabit H, Tauschmann M, Allen JM, et al. Home use of an artificial beta cell in type 1 diabetes. N Engl J Med 2015;373:2129-40. doi:10.1056/NEJMoa1509351

64 Garg SK, Weinzimer SA, Tamborlane WV, et al. Glucose outcomes with the in-home use of a hybrid closed-loop insulin delivery system in adolescents and adults with type 1 diabetes. Diabetes Technol Ther 2017:19:155-63 doi:10.1089/dia.2016.0421

65 Bekiari E, Karagiannis T, Haidich AB, Tsapas A. Meta-analysis of artificial pancreas trials: methodological considerations. Lancet Diabetes Endocrinol 2017;5:685. doi:10.1016/S22138587(17)30261-9

66 Agiostratidou G, Anhalt H, Ball D, et al. Standardizing clinically meaningful outcome measures beyond $\mathrm{HbA}_{1}$ for type 1 diabetes: a consensus report of the American Association of Clinical Endocrinologists, the American Association of Diabetes Educators, the American Diabetes Association, the Endocrine Society, JDRF International, The Leona M and Harry B Helmsley Charitable Trust, the Pediatric Endocrine Society, and the T1D Exchange. Diabetes Care 2017;40:1622-30. doi:10.2337/dc17-1624

67 Danne T, Nimri R, Battelino T, et al. International consensus on use of continuous glucose monitoring. Diabetes Care 2017:40:1631-40. doi: $10.2337 /$ dc17-1600

68 Maahs DM, Buckingham BA, Castle JR, et al. Outcome measures for artificial pancreas clinical trials: a consensus report. Diabetes Care 2016;39:1175-9. doi:10.2337/dc15-2716

69 Huyett L, Dassau E, Pinsker JE, Doyle FJ 3rd, Kerr D. Minority groups and the artificial pancreas: who is (not) in line? Lancet Diabetes Endocrinol 2016;4:880-1. doi:10.1016/S2213-8587(16)30144-9

70 Selph SS, Ginsburg AD, Chou R. Impact of contacting study authors to obtain additional data for systematic reviews: diagnostic accuracy studies for hepatic fibrosis. Syst Rev 2014:3:107. doi:10.1186/2046-4053-3-107
71 US Food and Drug Administration. The content of investigational device exemption (IDE) and premarket approval (PMA) applications for artificial pancreas device systems. Silver Spring, 2012.

72 Drazen JM, Morrissey S, Malina D, Hamel MB, Campion EW. The importance-and the complexities-of data sharing. N Engl I Med 2016;375:1182-3. doi:10.1056/NEJMe1611027

73 Taichman DB, Backus J, Baethge C, et al. Sharing clinical trial data: a proposal from the International Committee of Medical Journal Editors. IAMA 2016:315:467-8. doi:10.1001/jama.2015.18164

74 Bode BW, Johnson JA, Hyveled L, Tamer SC, Demissie M. Improved postprandial glycemic control with faster-acting insulin aspart in patients with type 1 diabetes using continuous subcutaneous insulin infusion. Diabetes Technol Ther 2017;19:25-33. doi:10.1089/ dia.2016.0350

75 Thabit H, Hartnell S, Allen JM, et al. Closed-loop insulin delivery in inpatients with type 2 diabetes: a randomised, parallel-group trial. Lancet Diabetes Endocrinol 2017;5:117-24. doi:10.1016/S22138587(16)30280-7

76 Barnard KD, Hood KK, Weissberg-Benchell J, Aldred C, Oliver N, Laffel L. Psychosocial assessment of artificial pancreas (AP): commentary and review of existing measures and their applicability in AP research. Diabetes Technol Ther 2015;17:295-300. doi:10.1089/dia.2014.0305

77 Barnard KD, Pinsker JE, Oliver N, Astle A, Dassau E, Kerr D. Future artificial pancreas technology for type 1 diabetes: what do users want? Diabetes Technol Ther 2015;17:311-5. doi:10.1089/ dia.2014.0316

78 Elleri D, Acerini CL, Allen JM, et al. Parental attitudes towards overnight closed-loop glucose control in children with type 1 diabetes. Diabetes Technol Ther 2010;12:35-9. doi:10.1089/ dia.2009.0084

79 van Bon AC, Brouwer TB, von Basum G, Hoekstra JB, DeVries JH. Future acceptance of an artificial pancreas in adults with type 1 diabetes. Diabetes Technol Ther 2011;13:731-6. doi:10.1089/ dia.2011.0013

Web appendix: Appendices 